\title{
Fractal Antenna Design for Overtaking on Highways in 5G Vehicular Communication Ad-hoc Networks Environment
}

\author{
Abdul Rahim, Praveen Kumar Mallik, V.A.Sankar Ponnapalli
}

\begin{abstract}
Vehicular Communication is designed for enhancing road safety and support autonomous driving becoming very popular in the present scenario. The proposed system aims to design a suitable and secured system for overtaking on highways. The main problem faced while driving vehicle on highways is to overtake a larger vehicle on narrow roads, where the driver visibility is limited and it is very risky to overtake from right side as there is a high probability of colliding with the vehicles coming in opposite direction. The proposed system solves the problem by establishing ad-hoc connection in $5 G$ environment with the vehicle to be overtaken. The system consists of a communication unit designed with a controller along with various options which are used to decide when the vehicle can safely overtake. In this way the driver can safely overtake the larger vehicles without any risk of road accidents. The proposed system uses fractal geometry to design the antenna which is used for the vehicle to vehicle communication at $5 G$ frequencies up to $20 \mathrm{GHz}$.
\end{abstract}

Keywords: vehicle to vehicle communication, Fractal Antenna, 5G, Ad-hoc networks, Controller, Antenna

\section{INTRODUCTION}

The goal of Vehicular Communication is to provide safety and comfort while driving on the roads. The present system consists of communication between vehicles to detect the acceleration and deceleration of nearby vehicles using ad-hoc network or using available communication network [1,6]. Long Term Evaluation (LTE) and dedicated short range communication (DSRC) are used for communicating between the vehicles using $3 \mathrm{G}$ network. The connection is established using the frequencies of $2.4 \mathrm{GHz}$ range end to end communication [2,3]. Intelligent transport system (ITS) uses ad-hoc network VANET when the communication network is offline [7]. The VANET uses network access points (APs) to establish connection between the vehicles for communication [8]. Existing systems uses any of the above communication which is limited in the terms of applications, security, efficiency, and connection establishment and safety measures [4]. The proposed system uses the frequencies in $5 \mathrm{G}$ spectrum to overcome the drawbacks of existing system [9-14].

The death rate due to road accidents are increasing every year worldwide, and many efforts are made to avoid the accidents and in this process this paper is one of the effort to avoid accidents while overtaking the vehicles. The proposed

\footnotetext{
Revised Manuscript Received on December 12, 2019

Abdul Rahim ,Research Scholar Lovely Professional University, Punjab, India, rahim.mrecw@gmail.com

Dr.Praveen Kumar Mallik ,Professor, Lovely Professional University, Punjab, India

Dr.V.A.Sankar Ponnapalli Shreyas Institute of Engineering \&Technology, Hyderabad
}

system aims to communicate with the vehicle in front to get the information related to safely overtake the vehicle[5].

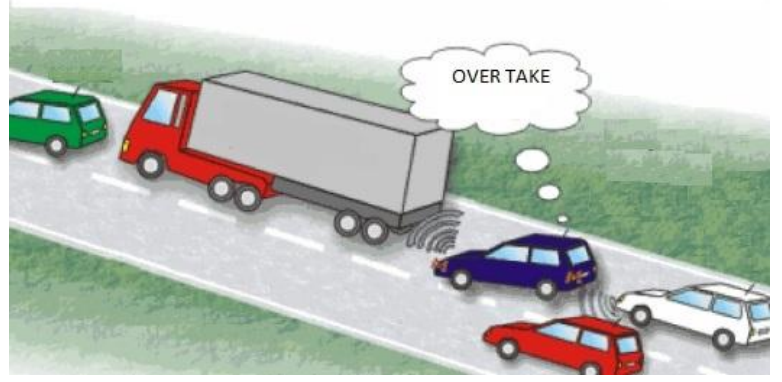

The rest of the paper is organized as follows. The proposed system is illustrated in section II. In section III, methodology is described. Applications, advantages, limitations and scope for future work are presented in section IV and finally concluding remarks are provided in section $\mathrm{V}$.

\section{PROPOSED SYSTEM}

The Proposed system consists of an 8-bit microcontroller which is the heart of the system. This microcontroller consist of a processor ATmega32u4 (Arduino Leonardo) with a 8-bit CPU working at the frequency of $16 \mathrm{MHz}$ clock speed. The micro controller consist of 20 digital I/O pins and 12 pins for connecting analog devices. As shown in the block diagram controller unit consists of two sub units, out of which one is a transmitter unit and the second is receiver unit.

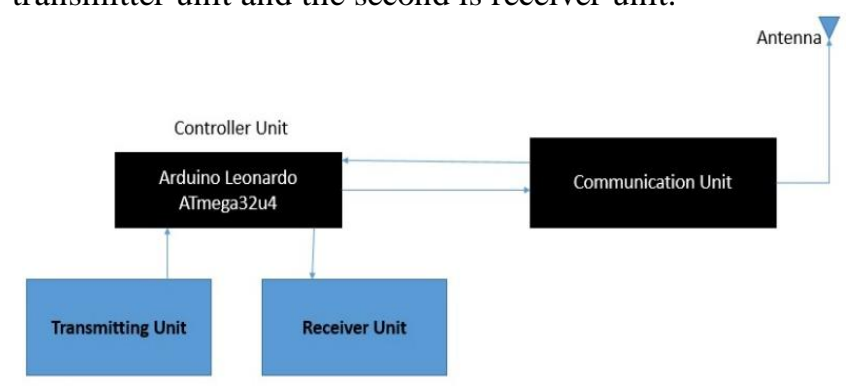

Transmitter unit consist of overtake switch which can be used when the vehicle needs to overtake. The driver uses this option which generates and interrupt to the controller. In turn the controller send the information to the communication unit which is used to transmit the request to the near-by vehicle using $5 \mathrm{G}$ ad-hoc network.

Receiver unit upon receiving the information from the $5 \mathrm{G}$ ad-hoc network, passes the information to the micro-controller which intern generates the control signals which are connected to the LED'S which indicates accept and reject options.

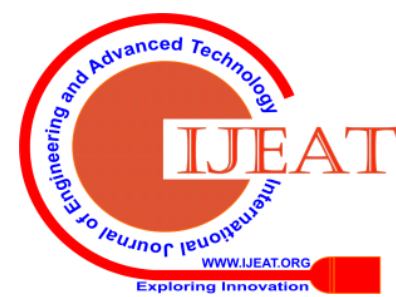


Along with controller unit, block diagram consist of communication unit which is used for communication between two vehicles in vehicular communication ad-hoc network. This unit works as a trans receiver which converts the micro controller signals into $5 \mathrm{G}$ signals while transmitting and vice versa at the receiver. Ad-hoc network discover the nearby service vehicles that are willing to collaborate with the request vehicle. Once the connection is established the request vehicle transmit the signals in the $5 \mathrm{G}$ range of frequencies. The connection remains available till any or both the vehicles select end of the session option.

\section{METHODOLOGY}

The System is divided into two segments, first is controller unit and second is the communication unit Controller Unit:

This unit is the heart of the system and is connected with all the required interfacing devices, which are helpful for communicating between the vehicles. The controller unit has transmitter and receiver unit along with a LCD display. The transmitting unit consists of switches which are as follows

1. Establish Communication

2. Request for Overtake

3. Accept

4. Reject

5. End the Communication

Considering vehicles $\mathrm{A}$ and $\mathrm{B}$, out of which $\mathrm{B}$ vehicle is a large and slow moving vehicle which is ahead of the A vehicle. A vehicle is the vehicle which needs to overtake; both the vehicles have the same five options as listed above. Vehicle A driver presses the first button which is for establishing the connection, this triggers the communication unit. The communication unit will be discussed later in the paper, once the communication is established; the driver of vehicle A now presses the second switch which is for requesting the vehicle $\mathrm{A}$ for overtake. As the request goes to the vehicle B, the driver of vehicle B either Accept the Request or Reject the Request. Once the information is passed to vehicle $A$, the vehicle A driver can end the communication by pressing the fifth switch.

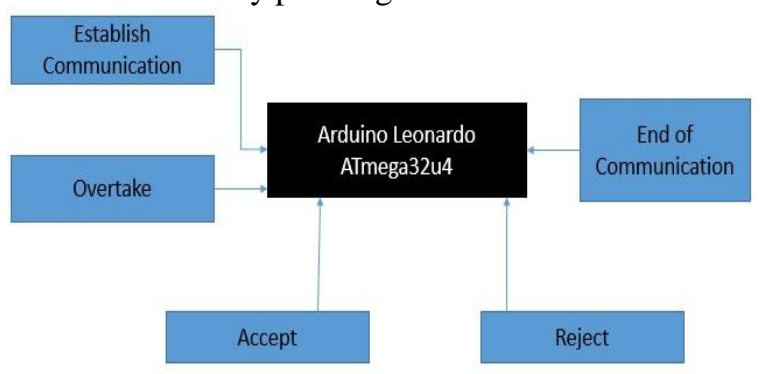

Transmitting Unit

The receiving unit consists of the following interface

1. Acknowledge Communication

2. LED's for Accept and Reject

As any vehicle needs to communicate, they send request as Establish Communication which intern vehicle $B$ acknowledge using the Acknowledge Communication such that vehicle to vehicle communication can be established. Once the communication is established the vehicle request for overtake, if overtake is possible the B vehicle will accept the request which can be seen on the vehicle A receiving unit where the accepted LED will be on. Once the Accepted LED is on, vehicle A can easily overtake the vehicle $\mathrm{B}$, and if it is rejected then Rejected LED will be on, that means at present it is very risky to overtake, upon which vehicle A will not overtake, and after some time once again vehicle A request for overtake, this process continues till the vehicle A overtakes Vehicle B Safely.

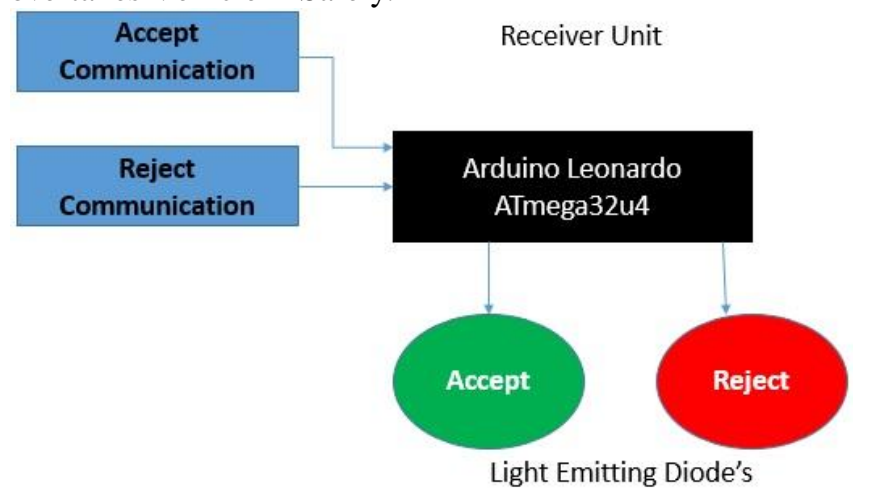

Fractal Antenna Design:

The Antenna is designed using fractal geometry which is widely used for wide band applications, and high beam width[16-21]. For the vehicular communication, this proposed antenna is very much suitable as high beam width is need for the application. The antenna is designed using the base of sierpinski square which is shown in the diagram below.

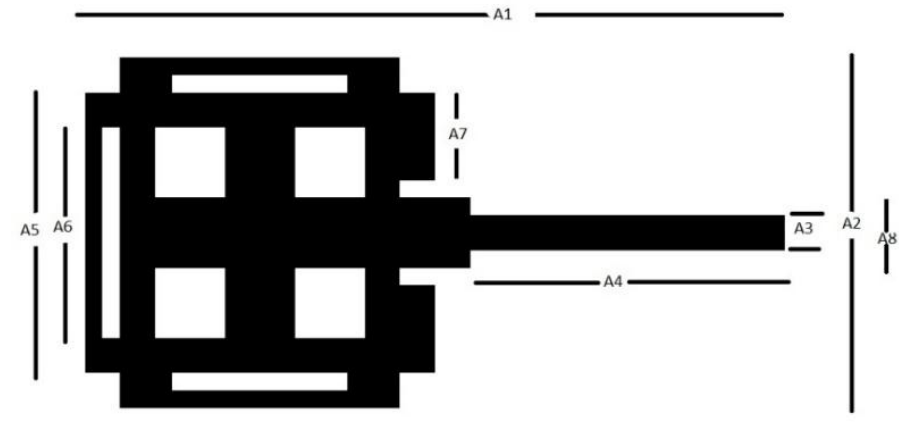

The dimensions for inner square is $2 \mathrm{mmX} 2 \mathrm{~mm}$ and the rectangles are $1 \mathrm{mmx} 2 \mathrm{~mm}$ and the remaining dimensions are provided below in the tabular column.

\begin{tabular}{|l|l|l|l|l|l|l|l|}
\hline A1 & A2 & A3 & A4 & A5 & A6 & A7 & A8 \\
\hline 30 & 24 & 2 & 12 & 20 & 16 & 4 & 3.5 \\
\hline
\end{tabular}

The below tabular column shows the VSWR for the frequencies ranging from $1 \mathrm{GHz}$ to $20 \mathrm{GHz}$. Resonant frequency is at $10 \mathrm{GHz}$ and the VSWR is around 50. 


\begin{tabular}{|c|c|c|}
\hline & Freq [GHz] & $\begin{array}{l}\text { dB(VSWR(1)) } \\
\text { Setup 1: Sweep }\end{array}$ \\
\hline 1 & 1.000000 & 55.808355 \\
\hline 2 & 2.000000 & 54.717943 \\
\hline 3 & 3.000000 & 53.246593 \\
\hline 4 & 4.000000 & 51.751593 \\
\hline 5 & 5.000000 & 50.540577 \\
\hline 6 & 6.000000 & 49.801748 \\
\hline 7 & 7.000000 & 49.567632 \\
\hline 8 & 8.000000 & 49.739092 \\
\hline 9 & 9.000000 & 50.157802 \\
\hline 10 & 10.000000 & 50.673742 \\
\hline 11 & 11.000000 & 51.176379 \\
\hline 12 & 12.000000 & 51.596388 \\
\hline 13 & 13.000000 & 51.895239 \\
\hline 14 & 14.000000 & 52.053270 \\
\hline 15 & 15.000000 & 52.059994 \\
\hline 16 & 16.000000 & 51.907548 \\
\hline 17 & 17.000000 & 51.590892 \\
\hline 18 & 18.000000 & 51.140068 \\
\hline 19 & 19.000000 & 50.738230 \\
\hline 20 & 20.000000 & 50.429563 \\
\hline & & \\
\hline
\end{tabular}

The below figure depicts the Radiation pattern for the proposed antenna and the radiation pattern is around 120 degrees which is very much useful for the vehicle to vehicle communication and its is having good directivity. The return losses are very much low which is very much helpful for radiating the entire energy towards forward direction. From the reported results it is clearly noticed that the proposed antenna will be suitable for real time vehicle to vehicle
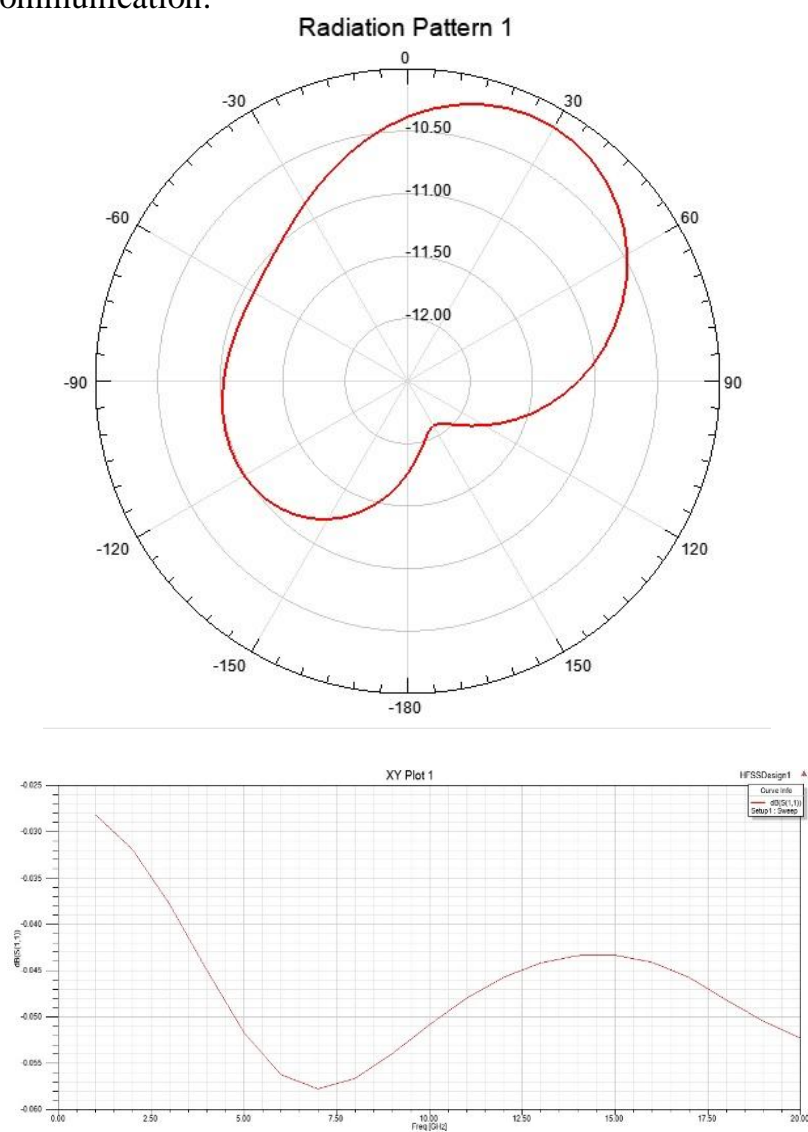
communication.

\section{APPLICATIONS}

Vehicle Safety: Highest Priority is given to the Vehicle safety while overtaking heavy vehicles on highways as many accidents are happening due to not having proper communication with the vehicles which are ahead travelling. This method can be used for overtaking the vehicles safely.

ADVANTAGES: Collision and Accidents avoidance: with the communication established between the vehicles, the collision and road accidents can be eliminated. Night time driving will be more comfortable with the proper communication between vehicles.

LIMITATIONS: Depending upon the vehicle, complexity of the systems and various interfacing devices the estimated cost of installing vehicle to vehicle communication would be around Rs.10, 000 to20, 000.

Scope for Future work: Along with Vehicle overtake, the system can introduce warning system when the vehicle comes very much near to other vehicles. A feedback system can be used for automatically applying the brakes when the vehicle is coming close to other vehicles. Automatically change the direction of vehicle when the system senses dividers.

\section{CONCLUSION}

The communication between the vehicles is used for enhancing the safety while driving on the high ways. The System designed uses 5G communication with Ad-hoc network for faster establishing the communication between the vehicles. The micro controller along with the interfacing devices are used to provide the information regarding overtaking the vehicles without any collision or accidents. Fractal antenna is used for wideband band application and the design is very much suitable for the vehicle to vehicle communication.

\section{REFERENCES}

1. Udit Narayana Kar, Debarshi Kumar Sanyal, An overview of device-to-device communication in cellular networks, ICT Express, Volume 4, Issue 4, 2018, Pages 203-208, ISSN 2405-9595, https://doi.org/10.1016/j.icte.2017.08.002.

2. T. Mondal, S. Maity, R. Ghatak and S. R. B. Chaudhuri, "Compact Circularly Polarized Wide-Beamwidth Fern-Fractal-Shaped Microstrip Antenna for Vehicular Communication," in IEEE Transactions on Vehicular Technology, vol. 67, no. 6, pp. 5126-5134, June 2018. doi: 10.1109/TVT.2018.2824841

3. F. Jameel, Z. Hamid, F. Jabeen, S. Zeadally and M. A. Javed, "A Survey of Device-to-Device Communications: Research Issues and Challenges," in IEEE Communications Surveys \& Tutorials, vol. 20, no. 3, pp. 2133-2168, thirdquarter 2018.doi: 10.1109/COMST.2018.2828120

4. P. Sondi, L. Rivoirard and M. Wahl, "Performance Evaluation of Vehicle-to-Vehicle Communications for a Collective Perception Application in Vehicular Ad hoc Networks," 2018 IEEE 29th Annual International Symposium on Personal, Indoor and Mobile Radio Communications (PIMRC), Bologna, 2018, pp. 602-603.doi: 10.1109/PIMRC.2018.8580753

5. Jameel, Furqan \& Wyne, Shurjeel \& Syed, Junaid \& Chang, Zheng. (2018). Propagation Channels for mmWave Vehicular Communications: State-of-the-art and Future Research Directions. .doi.10.1109/MWC.2018.1800174

6. M. Gholibeigi, N. Sarrionandia, M. Karimzadeh, M. Baratchi, H. van den Berg and G. Heijenk, "Reliable vehicular broadcast using 5G device-to-device communication," 2017 10th IFIP Wireless and Mobile Networking Conference (WMNC), Valencia, 2017, pp. 1-8.doi: 10.1109/WMNC.2017.824884 
7. Abdul Rahim, K Raghavendra, "Fractal Antenna Design for Various Multiband Applications", International Journal of Engineering and Advanced Technology (IJEAT) ISSN: 2249 - 8958, Volume-8, Issue-6S3, September 2019.

8. B.T.P. Madhav, T. Anilkumar, Sarat K. Kotamraju, Transparent and conformal wheel-shaped fractal antenna for vehicular communication applications,AEU - International Journal of Electronics and Communications, Volume 91,2018,Pages $\quad 1-10$, ISSN 1434-8411,https://doi.org/10.1016/j.aeue.2018.04.028.

9. H. Peng, Le Liang, X. Shen and G. Y. Li, "Vehicular Communications: A Network Layer Perspective," in IEEE Transactions on Vehicular Technology, vol. 68, no. 2, pp. 1064-1078, Feb. 2019.doi: 10.1109/TVT.2018.2833427

10. Bastien Béchadergue, Wen-Hsuan Shen, Hsin-Mu Tsai, Comparison of OFDM and OOK modulations for vehicle-to-vehicle visible light communication in real-world driving scenarios, Ad Hoc Networks, Volume $94,2019,101944$, ISSN 1570-8705,https://doi.org/10.1016/j.adhoc.2019.101944.

11. Malik P.K., Parthasarthy H., Tripathi M.P. (2013) Alternative Mathematical Design of Vector Potential and Radiated Fields for Parabolic Reflector Surface. In: Unnikrishnan S., Surve S., Bhoir D. (eds) Advances in Computing, Communication, and Control. ICAC3 2013. Communications in Computer and Information Science, vol 361. Springer, Berlin, Heidelberg

12. Aljawharah Alnasser, Hongjian Sun, Jing Jiang,Cyber security challenges and solutions for V2X communications: A survey,Computer Networks,Volume 151,2019,Pages 52-67,ISSN 1389-1286,https://doi.org/10.1016/j.comnet.2018.12.018.

13. Navjot Kaur, Sandeep Kad, A Review on Security Related Aspects in Vehicular Adhoc Networks, Procedia Computer Science,Volume 78,2016,Pages 387-394,ISSN 1877-0509, https://doi.org/10.1016/j.procs.2016.02.079.

14. C. Navaneethan, S. Meenatchi, Mutyala V.S. Rathnakumari, V. Thamaraiselvi, An Optimistic Approach for Data Retrieval in Vehicular Adhoc Networks, Procedia Computer Science, Volume 50, 2015,Pages 380-387,ISSN 1877-0509, https://doi.org/10.1016/j.procs.2015.04.031.

15. Ba Cao Nguyen, Tran Manh Hoang, Le The Dung,Performance analysis of vehicle-to-vehicle communication with full-duplex amplify-and-forward relay over double-Rayleigh fading channels, Vehicular Communications, Volume 19,2019,100166,ISSN 2214-2096,https://doi.org/10.1016/j.vehcom.2019.100166.

16. J. Saini and S. K. Agarwal, "Design a single band microstrip patch antenna at $60 \mathrm{GHz}$ millimeter wave for 5G application," 2017 International Conference on Computer, Communications and Electronics (Comptelix), Jaipur, 2017, pp. 227-230. doi: 10.1109/COMPTELIX.2017.8003969

17. Manisha Gupta, Vinita Mathur, Sierpinski fractal antenna for internet of things applications, Materials Today: Proceedings,Volume 4, Issue 9,2017,Pages 10298-10303,ISSN 2214-7853, https://doi.org/10.1016/j.matpr.2017.06.368.

18. Guru Prasad Mishra, Madhu Sudan Maharana, Sumon Modak, B.B. Mangaraj,

19. Study of Sierpinski Fractal Antenna and Its Array with Different Patch Geometries for Short Wave Ka Band Wireless Applications, Procedia Computer Science, Volume 115,2017,Pages 123-134,ISSN 1877-0509,https://doi.org/10.1016/j.procs.2017.09.085.

20. D. H. Werner and S. Ganguly, "An overview of fractal antenna engineering research," in IEEE Antennas and Propagation Magazine, vol. 45, no. 1, pp. 38-57, Feb. 2003.doi: 10.1109/MAP.2003.1189650.

21. Malik, P., \& Parthasarthy, H. . "Synthesis of randomness in the radiated fields of antenna array". International Journal of Microwave and Wireless Technologies, 3(6), 701-705,2011.

22. De, Arnab \& Chosh, C. \& Bhattacherjee, A.. (2016). Design and Performance Analysis of Microstrip Patch Array Antennas with different configurations. International Journal of Future Generation Communication and $\quad$ Networking. 9 97-110. 10.14257/ijfgen.2016.9.3.10

\section{AUTHORS PROFILE}

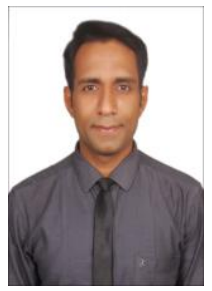

Abdul Rahim He is pursuing his $\mathrm{PhD}$ in department of Electronics and Communication at LOVELY PROFESSIONAL UNIVERSITY, Punjab, INDIA. He received his B.E in Electronics and Communication from Osmania University and M.Tech in VLSI system design for JNTUH. His current research interest include Vehicular Communication Applications, Fractal Antenna Design at milli meter frequencies. e-mail: rahim.mrecw@gmail.com

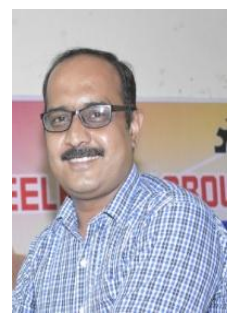

Dr. Praveen Kumar Malik A professional qualified and experienced person with extensive knowledge and skills in Antenna and Embedded System. He is working as Professor in the department of Electronics and Communication, Lovely Professional University, Punjab INDIA. He is B Tech, M Tech and Ph.D. from Electronics and Communication system. His major area of interest is signal processing, Antenna, and Embedded systems. He is having more than 10 papers in international refereed journals. Having more than 10 papers in the international and national conference also.

e-mail:pkmalikmeerut@gmail.com

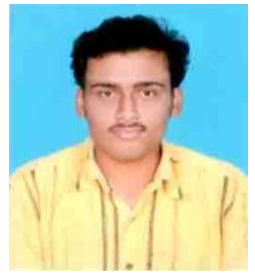

V. A. Sankar Ponnapalli He received his B.Tech in Electronics \& Communication Engineering from JNT University Kakinada in 2011, M. Tech in RF \& Microwave Engineering and the $\mathrm{PhD}$ degrees from GITAM (Deemed to be University) Visakhapatnam in 2013 and 2018 respectively. He is currently working as an Associate Professor in the Department of Electronics and Communication Engineering, Sreyas Institute of Engineering and Technology Hyderabad. He has authored or co-authored books, book chapters, and more than 25 research articles. He is acting as a reviewer and editorial board member for various international journals and conferences. His research interests include microwaves, antennas, and intelligent transportation systems etc. email id: vadityasankar3@gmail.com 